\title{
Kompetenznetz Schizophrenie
}

S chizophrenie zählt zu den schwersten und mit einer Lebenszeitprävalenz von etwa $1 \%$ zudem zu vergleichsweise häufig auftretenden psychischen Störungen. Obwohl mittlerweile gute Behandlungsmöglichkeiten für die meisten Patienten zur Verfügung stehen, ist die Erkrankung für die Betroffenen noch immer mit erheblichem persönlichen Leid und Stigmatisierung verbunden. So kommt es bei etwa einem Drittel der Ersterkrankten trotz Therapie zu deutlichen Einschränkungen im alltäglichen Leben mit Erwerbsunfähigkeit, gesellschaftlicher Ausgrenzung und sozialer Isolierung. Schizophrenie rechnet zu den zehn häufigsten zu einer Behinderung führenden Erkrankungen. Etwa 10-15\% der Patienten begehen Suizid. Aufgrund der Notwendigkeit zu häufiger und langer Behandlung, zu aufwändigen Rehabilitations- und Betreuungsmaßnahmen sowie insbesondere aufgrund der hohen Erwerbsunfähigkeit ist Schizophrenie jedoch nicht nur eine große Belastung für die Erkrankten und ihre Angehörigen, sondern auch für die Gesellschaft. Schizophrenie rechnet zu den teuersten psychischen Erkrankung, ihre direkten und indirekten Kosten sind mit denen somatischer Volkskrankheiten wie Diabetes oder Herzerkrankungen vergleichbar.

Eine frühzeitigere Erkennung der Erkrankung und eine vollständigere Ausnutzung der bestehenden Behandlungs- und Rehabilitationsmöglichkeiten können die Aussichten auf einen günstigen Krankheitsverlauf allerdings beträchtlich erhöhen. Die Optimierung der Früherkennung und -behandlung, der Akut- und Langzeittherapie sowie der Rehabilitation schizophrener Störungen sind daher primäre Zielsetzungen des bundesweiten Forschungsverbunds „Kompetenznetz Schizophrenie“", der seit 1999 vom Bundesministerium für Bildung und Forschung (BMBF) gefördert wird. Zur Umsetzung dieser Ziele werden im Kompetenznetz Schizophrenie unter Kooperation von etwa 50 Forschungsinstitutionen, Kliniken der Routineversorgung und Praxenverbünden annähernd 30 inhaltlich und methodisch eng aufeinander abgestimmte Forschungsprojekte durchgeführt (www.kompetenznetz-schizophrenie.de).

Das Netz zählt zu den neun ersten von inzwischen 17 Kompetenznetzen in der Medizin, die jeweils krankheitsspezifische Kompetenzen aus Forschung und Versorgung zusammenführen sollen, um der Forschung unmittelbare Impulse aus der Versorgung zu geben und neue Forschungsergebnisse rascher in die Versorgungspraxis zu transferieren. Hierzu sollen langfristige Kommunikations- und Kooperationsstrukturen etabliert werden, wobei auch die Verbände des Gesundheitswesens, medizinische Fachgesellschaften, Kostenträger, Betroffene, Angehörige und pharmazeutische Unternehmen einbezogen werden. Daher sollen die Kernstrukturen des Netzes auch nach Ablauf der öffentlichen Förderung aufrecht erhalten bleiben. Bereits im September 2000 ist aus diesem Grund der gemeinnützige Verein „PsychoseNetz e.V.“ (www.psychosenetz.de) gegründet worden, der sowohl einzelne Projekte als auch die Öffentlichkeitsarbeit des Netzes fördern soll. Nachdem die vom Kompetenznetz seit dem Jahr 2000 durchgeführten Langzeitstudien im Jahr 2005 abgeschlossen werden, soll die kürzlich vom BMBF bewilligte letzte Förderperiode bis zum Jahr 2008 insbesondere dazu dienen, den Transfer der Forschungsergebnisse in die Versorgung zu befördern und die strukturellen Grundlagen des Kompetenznetzes zu festigen. Daneben wird das Kompetenznetz selbstverständlich auch weiterhin die Koordination und Durchführung von klinischen Studien wahrnehmen, die dann jedoch mit unabhängig vom BMBF einzuwerbender Förderung durchzuführen sind.

Inhaltlich sieht das Kompetenznetz Schizophrenie dabei weiterhin seinen Schwerpunkt in der Entwicklung von zielgruppen- und praxisorientierten Angeboten aus den Bereichen der Früherkennung und Frühintervention und der Akut- und Langzeitbehandlung von Ersterkrankten sowie in der Verbesserung der psychiatrischen Versorgung durch Qualitätsmanagementstrategien und dem Abbau von Stigma und Diskriminierung durch zielgruppenspezifische Aufklärung.

Das vorliegende Themenheft Schizophrenie greift diese Schwerpunkte auf und stellt den aktuellen Kenntnisstand sowie die zum jeweiligen Themenbereich bisher in den - überwiegend noch laufenden - Forschungsprojekten des Kompetenznetzes gewonnenen Erkenntnisse dar. Sofern Sie an weiteren Informationen oder an einer Mitarbeit im Kompetenznetz interessiert sind, steht Ihnen die an der Psychiatrischen Universitätsklinik Düsseldorf, Rheinische Kliniken Düsseldorf angesiedelte Netzwerkzentrale gerne zur Verfügung (info@kompetenznetzschizophrenie.de).

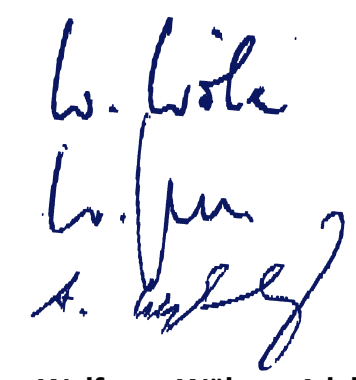

Wolfgang Wölwer, Adelheid Weßling, Wolfgang Gaebel Kompetenznetz Schizophrenie 